\title{
Aspirin inhibits endometrial fibrosis by suppressing the TGF-ß1-Smad2/Smad3 pathway in intrauterine adhesions
}

\author{
ZIHUI ZHANG ${ }^{1,2^{*}}$, SHUANG LI ${ }^{1,2^{*}}$, JIE DENG $^{1,2}$, SHAORONG YANG $^{3}$, ZIWU XIANG $^{3}$ \\ HONGYAN GUO $^{1}$, HONGLI XI ${ }^{1}$, MING SANG $^{1}$ and WEI ZHANG ${ }^{1}$ \\ ${ }^{1}$ Department of Obstetrics and Gynaecology, ${ }^{2}$ Laboratory of Gynaecological Oncology and \\ Reproductive Health, ${ }^{3}$ Department of Pathology, Xiangyang No. 1 People's Hospital, \\ Hubei University of Medicine, Xiangyang, Hubei 441000, P.R. China
}

Received August 28, 2019; Accepted January 24, 2020

DOI: $10.3892 /$ ijmm.2020.4506

\begin{abstract}
Intrauterine adhesions (IUAs) represent one of the most common diseases in women of reproductive age. Patients with moderate-to-severe IUA can experience a decrease in normal menstrual patterns, amenorrhea and even infertility. At present, the first-line treatment strategies for IUAs in the clinical practice are hysteroscopic transuterine resection of adhesion and postoperative adjuvant therapy, including oestrogen. However, a high recurrence rate of IUAs remains. In recent years, studies have demonstrated that aspirin combined with oestrogen may significantly prevent the postoperative disease recurrence rate, improve endometrial receptivity and improve the conception rate by increasing endometrial blood supply and angiogenesis more effectively. The TGF- $\beta 1-S m a d 2 / S m a d 3$ pathway is one of the important mechanisms involved in endometrial fibrosis. However, whether aspirin can inhibit endometrial fibrosis through the TGF- $\beta 1$-Smad2/Smad3 pathway to prevent postoperative re-adhesion remains to be elucidated. The results of the present study suggested that aspirin inhibits endometrial fibrosis by suppressing the TGF- $\beta 1-$ Smad2/Smad3 pathway, which may provide new hypotheses for the mechanism of action of aspirin in the treatment of IUAs.
\end{abstract}

\section{Introduction}

Intrauterine adhesions (IUAs) were first defined by Joseph Asherman in 1948, and their presence is also known as

Correspondence to: Dr Wei Zhang, Department of Obstetrics and Gynaecology, Xiangyang No. 1 People's Hospital, Hubei University of Medicine, 15 Jiefang Avenue, Xiangyang, Hubei 441000, P.R. China

E-mail: zhangwei_xyyy@126.com

${ }^{*}$ Contributed equally

Key words: aspirin, oestradiol valerate, endometrial fibrosis, intrauterine adhesions
Asherman syndrome (1). Endometrial fibrosis is caused by various factors, such as trauma and infection, and it results in impaired endometrial function, endometrial adhesion, uterine cavity degeneration and progression to an IUA $(2,3)$. The primary clinical symptoms of IUAs include decreased menstruation, amenorrhea, repeated spontaneous abortion and infertility (3), which have adverse effects on the physical and mental health of the patients. For infertility patients who wish to remain fertile, moderate-to-severe IUAs require surgery (3). Hysteroscopic transuterine resection of adhesion (TCRA) is the most important and commonly used treatment for moderate-to-severe intrauterine adhesions. TCRA has a significant role in improving menstruation and increasing pregnancy rate (4), but the disease recurrence rate following TCRA is $>62 \%$ (5). Therefore, the long-term treatment results remain unsatisfactory. The prevention and decrease recurrence following TCRA is a major clinical problem.

Transforming growth factor- $\beta 1$ (TGF- $\beta 1$ ) serves as a multifunctional cellular regulator. Following activation of TGF- $\beta 1$, activated TGF- $\beta 1$ binds to its receptor and activates downstream Smad signalling, including Smad2 and Smad3, which serves a crucial role in tissue fibrosis $(6,7)$, including cardiac (8), liver (9), pulmonary (10), pancreatic (11) and renal fibrosis (12), and chronic autoimmune disease Sjögren's syndrome (SS) (13). The mechanism of the TGF- $\beta 1 / \mathrm{Smad}$ signalling pathway involved in the occurrence of intrauterine adhesions has been described previously $(14,15)$.

Aspirin is a non-steroidal anti-inflammatory drug whose primary active component is acetylsalicylic acid (16). Aspirin is clinically used to decrease fever, inflammation, cardiovascular disease and certain types of cancer (17-19). Jiang et al (20) described the association between the use of aspirin and liver fibrosis in 1,856 patients with chronic liver disease in the USA. The results revealed that the liver fibrosis index in patients using aspirin was decreased compared with those who did not use aspirin. A recent study in a rat liver fibrosis model demonstrated that aspirin may significantly improve the degree of liver fibrosis in rats (21). In addition, aspirin has a positive effect on improving cardiac fibrosis (22). A recent study has demonstrated that aspirin has a positive effect on the growth and repair of the endometrium following IUAs (23), suggesting that this may be associated with the promotion 
of endometrial microvascular formation and improvement of local blood circulation by aspirin, thereby decreasing IUA recurrence, improving menstruation and increasing the pregnancy rate (23). To the best of our knowledge, there are no previous studies investigating whether aspirin inhibits endometrial fibrosis by inhibiting the TGF- $\beta 1-\mathrm{Smad} 2 / \mathrm{Smad} 3$ pathway and decreases postoperative recurrence of IUAs.

\section{Materials and methods}

Patient selection. The present study recruited 54 patients with IUAs who were admitted to the Xiangyang No. 1 People's Hospital, Hubei University of Medicine between July 2018 and July 2019. The present study was reviewed and approved by the Ethics Committee of Xiangyang No. 1 People's Hospital, Hubei University of Medicine (approval no. 2018KYLL). All patients provided written informed consent prior to the study. The inclusion criteria were: Patients diagnosed with IUAs by hysteroscopy; patients with a history of infertility who wished to become pregnant; and patients who were reviewed again with good compliance. The exclusion criteria were: Infection; other diseases of the uterus; hormone-dependent or malignant diseases; and patients who received hormone therapy within 3 months prior to surgery. All patients underwent hysteroscopic investigation of the IUAs within 3-7 days following the end of the menstruation cycle. The IUA scores and grades were evaluated according to the revised criteria of the American Fertility Association (AFS) (24).

Postoperative artificial menstrual cycle therapy and follow-up. Patients with IUAs were randomly divided into two groups. All patients underwent TCRA surgery and received oral medication. Patients in group A (observation group; $\mathrm{n}=26$ ) were given $4 \mathrm{mg}$ /day oestradiol valerate for 21 days, and $1 \mathrm{mg} /$ day cyproterone acetate was given for the last 10 of the 21 days for artificial cycle therapy for a total of 2 cycles. Group B (combination therapy group; $n=28$ ) received $100 \mathrm{mg} /$ day aspirin and $4 \mathrm{mg} /$ day oestradiol valerate for 21 days, and $1 \mathrm{mg} /$ day cyproterone acetate was given during the last 10 of the 21 days for artificial cycle therapy for a total of 2 cycles. Patients were treated continuously for 2 months. All patients from both groups received TCRA and the placement of an intrauterine-suitable balloon in the uterus for 1 week. During the postoperative follow-up examinations, there was no postoperative infection or abdominal pain observed in any of the patients. The outcomes of the 2 different therapies after 2 months were assessed using the following indicators: Uterine length, endometrial thickness, menstrual flow and volume, postoperative adhesion cases and postoperative adhesion score according to the AFS standard. Hysteroscopy was performed by the same senior doctor at the time of admission and 2 months following surgery.

Histological staining, masson trichrome staining and immunohistochemistry (IHC). The endometrial tissues were fixed in $4 \%$ formalin for a minimum of $24 \mathrm{~h}$. The fixed tissues were embedded in paraffin and cut to $4-\mu \mathrm{m}$ thick sections for staining. The tissues were stained using a Masson's trichrome staining according to the manufacturer's protocol (cat. no. G1345; Beijing Solarbio Science \& Technology Co., Ltd.).
The sections were immersed in bouin buffer, incubated at $37^{\circ} \mathrm{C}$ for $2 \mathrm{~h}$ and rinsed three times with PBS. Samples were then treated with the following agents at room temperature, with a rinse in warm running water for 3 min after each application: $0.5 \%$ celestine blue staining solution for $3 \mathrm{~min}$; Mayer (1\%) hematoxylin staining solution for $3 \mathrm{~min}$; and $1 \%$ acidic ethanol differentiation solution for $10 \mathrm{sec}$. The following agents were then administered, with a triplicate wash with distilled water between: $1 \%$ acid fuchsin solution staining solution for $10 \mathrm{~min} ; 1 \%$ phosphomolybdic acid solution for $10 \mathrm{~min}$; and $2 \%$ aniline blue solution for $5 \mathrm{~min}$. After dehydration with 95\% ethanol samples were treated with absolute ethyl alcohol in triplicate for a duration of $10 \mathrm{sec}$ per treatment. Finally, samples were treated with $50 \%$ xylene solution for $2 \mathrm{~min}$ and $100 \%$ xylene solution for $2 \mathrm{~min}$. The percentage of blue staining indicated the extent of endometrial fibrosis. For IHC (according to the manufacturer's protocol of the immunohistochemical staining kit; cat. no. E670016, Sangon Biotech Co., Ltd.), tissue paraffin sections were deparaffinized in xylene (100\% for $10 \mathrm{~min} ; 50 \%$ for $10 \mathrm{~min}$ ) and rehydrated using an alcohol gradient $(100 \%$ for $5 \mathrm{~min}$; $95 \%$ for $5 \mathrm{~min} ; 70 \%$ for $5 \mathrm{~min}$ ) at room temperature. Antigen retrieval was performed by heating in a high-pressure induction cooker for $100 \mathrm{sec}$, and endogenous peroxidase activity was quenched by immersing the samples in $3 \% \mathrm{H}_{2} \mathrm{O}_{2}$ for $10 \mathrm{~min}$. Samples were then incubated with anti-rabbit TGF- $\beta 1(20 \mu \mathrm{g} / \mathrm{ml}$; cat. no. ab92486; Abcam), anti-rabbit phosphorylated (p)-Smad2 (1:100; cat. no. ab53100; Abcam), anti-rabbit p-Smad3 (1:100; cat. no. ab74062; Abcam), anti-rabbit $\alpha$-smooth muscle actin ( $\alpha$-SMA; 1:100; cat. no. ab32575; Abcam), anti-rabbit collagen type I $\alpha 1$ chain $(\mathrm{COL} 1 \mathrm{~A} 1 ; 1 \mu \mathrm{g} / \mathrm{ml}$; cat. no. LS-C343921; Lifespan BioSciences, Inc.) and anti-rabbit fibronectin (FN; 1:200; cat. no. ab2413; Abcam) primary antibodies overnight at $4^{\circ} \mathrm{C}$. The slides were washed with PBS and incubated with normal goat serum (1:50; cat. no. A0208; Beyotime Institute of Biotechnology) at $37^{\circ} \mathrm{C}$ for $15 \mathrm{~min}$. The slides were then stained with $2 \%$ 3,3-diaminobenzidine, and cell nuclei were stained with $1 \%$ haematoxylin at room temperature for $3 \mathrm{~min}$. Images were captured in randomly selected fields of each section at magnification, $\mathrm{x} 200$, and used for analysis of the positive staining. The results of the Masson trichrome staining and IHC were evaluated quantitatively using the ImageJ software [v.1.4.3.67; National Institutes of Health (NIH)]. Average optical density (AOD) was used in the present study for the statistical analysis and was calculated using the following formula: $\mathrm{AOD}=$ integrated optical density (IOD)/area.

Reverse transcriptionquantitative polymerase chain reaction ( $R T-q P C R)$ assay. The total RNA of endometrium tissues was isolated using a High PureRNA Isolation kit (Invitrogen; Thermo Fisher Scientific, Inc.) according to the manufacturer's protocol. The amount of total RNA was estimated by measuring the absorbance at 260 and $280 \mathrm{~nm}$, and the purity of each sample was determined according to the A260/A280 ratios using a SMA2000 spectrophotometer (Thermo Fisher Scientific, Inc.). A260/A280 ratio values of $\geq 1.8$ and $\leq 2$ were used an indicator of good quality RNA. For detection of the $\alpha$-SMA, COL1A1 and FN mRNA levels, $1 \mu \mathrm{g}$ total RNA was reverse transcribed at $37^{\circ} \mathrm{C}$ for $60 \mathrm{~min}$ using the miRcute miRNA First-Strand cDNA Synthesis kit 
Table I. Sequences of the reverse transcription quantitative polymerase chain reaction primers used.

\begin{tabular}{ll}
\hline Name & \\
\hline$\alpha$-SMA & \multicolumn{1}{c}{ Sequence } \\
COL1A1 & Forward: 5'-GCTTCCTCTTCTTCCCTGGAG-3' \\
& Reverse: 5'-AGATGGCTGGAAGAGGGTCTC-3' \\
FN & Forward: 5'-GGCATAAAGGGTCATCGTG-3' \\
& Reverse: 5'-GAACCTTCGCTTCCATACTC-3' \\
GAPDH & Forward: 5'-GAGAGATCTGGAGGTCAT-3' \\
& Reverse: 5'-GGGTGACACCTGAGTTGAA-3' \\
& Forward: 5'-CGGAGTCAACGGATTTGGTCGTAT-3' \\
& Reverse: 5'-AGCCTTCTCCATGGTGGTGAAGAC-3'
\end{tabular}

$\alpha$-SMA, $\alpha$-smooth muscle actin; COL1A1, collagen type I $\alpha 1$ chain; FN, fibronectin.

(Beyotime Institute of Biotechnology) and then amplified using an Applied Biosystems 7900HT cycler using SuperReal PreMix Plus (Beyotime Institute of Biotechnology). The qPCR thermocycling conditions were as follows: Predenaturation at $94^{\circ} \mathrm{C}$ for $5 \mathrm{~min}, 40$ cycles of denaturation at $94^{\circ} \mathrm{C}$ for $20 \mathrm{sec}$ and annealing at $60^{\circ} \mathrm{C}$ for $20 \mathrm{sec}$. GAPDH was used as the normalization control for qPCR. The relative expression was calculated using the $2^{-\Delta \Delta \mathrm{Cq}}$ method (25). Each reaction was performed in triplicate. The primers used for the RT-qPCR analysis are presented in Table I.

Western blot analysis. Endometrial tissues were lysed with a radio immune precipitation assay buffer containing protease inhibitors. Proteins were quantified using a BCA protein assay kit. Aliquots of the lysates $(30 \mu \mathrm{g})$ were separated via $10 \%$ SDS-PAGE, transferred onto nitrocellulose membranes and blocked with 5\% skimmed milk at room temperature for $2 \mathrm{~h}$. Primary antibodies against TGF- $\beta 1$ (4 $\mu \mathrm{g} / \mathrm{ml}$; cat. no. ab92486; Abcam), anti-rabbit p-Smad2 (1:500; cat. no. ab53100; Abcam), anti-rabbit Smad2 (1:2,000; cat. no. ab40855; Abcam), anti-rabbit p-Smad3 (1:500; cat. no. ab74062; Abcam), anti-rabbit Smad3 (1:2,000; cat. no. ab40854; Abcam), anti-rabbit $\alpha$-SMA (1:2,000; cat. no. ab32575; Abcam), anti-rabbit COL1A1 $(0.1 \mu \mathrm{g} / \mathrm{ml}$; cat. no. LS-C343921; Lifespan BioSciences, Inc.); anti-rabbit fibronectin (1:500; cat. no. ab2413; Abcam); and anti-rabbit GAPDH (1:500; Beyotime Institute of Biotechnology) were used in the analysis. The membranes were incubated with horseradish peroxidase-conjugated goat anti-rabbit IgG (1:1,000; cat. no. A0208; Beyotime Institute of Biotechnology) for $1 \mathrm{~h}$ at $37^{\circ} \mathrm{C}$. Enhanced chemiluminescence (Thermo Fisher Scientific, Inc.) was used to visualize the bands. ImageJ software (v.1.4.3.67; NIH) was used to quantify the intensity of the bands, using GAPDH as the control.

Statistical analysis. Measurement data are expressed as the mean \pm standard deviation. Normally distributed variables (average age, body mass index and times of labour) were compared using Student's t-test between 2 groups, and the Mann-Whitney U test were used for non-normally distributed variables (times of pregnancy, surgical abortion, postoperative average AFS score) between 2 groups. All datasets in which both pre- vs. postoperative and group A vs. group B comparisons were statistically compared by two-way repeated-measures ANOVA, and a Bonferroni's post hoc test was used to correct for multiple comparisons. Count data were expressed as the number of cases as a percentage. Comparisons between groups were performed using a $\chi^{2}$ test or Fisher's exact test. $\mathrm{P}<0.05$ was considered to indicate a statistically significant difference. All statistical analyses were performed using SPSS (v.22.0; IBM Corp.)

\section{Results}

Demographic data in the study groups. The baseline demographics and clinical characteristics, including age, body mass index, times of pregnancy, times of labour, IUA grade and most probable aetiology of IUA, of the 54 patients included in the present study were not significantly different (Table II).

Patients with IUA receiving a combination of oestradiol valerate and aspirin therapy exhibit better effects than those with simple oestradiol valerate therapy. The results revealed that the recurrence rate of postoperative adhesion and average AFS score in the combination therapy group was significantly decreased compared with those in the oestradiol valerate-alone therapy group (Table II). In both treatment groups, significant differences in uterine length, menstrual flow, menstrual volume and endometrial thickness between the pre- and postoperative values within the same group were observed. In addition, the combination therapy group exhibited greater improvements, including duration of menstrual period (4.50 \pm 0.71 days in group $\mathrm{A}$ vs. $5.39 \pm 1.20$ days in group $\mathrm{B} ; \mathrm{P}<0.01)$, menstrual volume $(55.77 \pm 8.45 \mathrm{ml}$ in group $\mathrm{A}$ vs. $63.57 \pm 12.39 \mathrm{ml}$ in group $\mathrm{B} ; \mathrm{P}<0.01)$, endometrial thickness $(6.88 \pm 0.77 \mathrm{~mm}$ in group A vs. $7.54 \pm 1.14 \mathrm{~mm}$ in group $\mathrm{B}$; $\mathrm{P}<0.05)$, no adhesion cases following operation $[34.6 \%(9 / 26)$ in group A vs. $75.0 \%$, $21 / 28$ in group $\mathrm{B} ; \mathrm{P}<0.05$ ] and postoperative average AFS score $(5.62 \pm 2.42$ in group A vs. $3.43 \pm 1.73$ in group $\mathrm{B} ; \mathrm{P}<0.01)$, with the exception of uterine length $(7.09 \pm 0.22 \mathrm{~cm}$ in group $\mathrm{A}$ vs. $7.13 \pm 0.26 \mathrm{~cm}$ in group $\mathrm{B}(\mathrm{P}=0.253)$ (Table III).

Combination therapy with oestradiol valerate and aspirin inhibits endometrial fibrosis in patients with IUA. Endometrial fibrosis was measured in the tissues with 
Table II. Demographic data of the study groups.

\begin{tabular}{lcc}
\hline Variable & $\begin{array}{c}\text { Group A } \\
(\mathrm{n}=26)\end{array}$ & $\begin{array}{c}\text { Group B } \\
(\mathrm{n}=28)\end{array}$ \\
\hline Average age, years & $28.15 \pm 3.68$ & $29.07 \pm 4.25$ \\
Body mass index & $22.16 \pm 2.18$ & $22.01 \pm 1.99$ \\
Times of pregnancy & $3.81 \pm 1.13$ & $3.96 \pm 1.07$ \\
Times of labour & $0.88 \pm 0.82$ & $1.01 \pm 0.79$ \\
IUA grade (n) & & \\
$\quad \begin{array}{l}\text { Moderate } \\
\text { Severe }\end{array}$ & $15(57.7)$ & $16(57.1)$ \\
$\begin{array}{l}\text { Most probable etiology of IUA } \\
\text { Surgical abortion (average times) }\end{array}$ & $2.81 \pm 1.33$ & $2.86 \pm 1.27$ \\
$\begin{array}{l}\text { Curettage in the middle and late } \\
\text { pregnancy (\%) }\end{array}$ & $4(15.4)$ & $3(10.7)$ \\
Pelvic inflammatory disease (\%) & $1(3.8)$ & $2(7.1)$ \\
Other etiology & No & No \\
\hline
\end{tabular}

Data are presented as the mean \pm standard deviation or $\mathrm{n}(\%)$. Group A, oestradiol valerate therapy; Group B, combination therapy of aspirin and oestradiol valerate; IUA, intrauterine adhesion.

Masson's trichrome staining. The results revealed that the endometrial tissues from the two postoperative groups exhibited less fibrosis compared with the tissues from the preoperative groups. However, the combined group exhibited significantly decreased levels of endometrial fibrosis compared with the oestradiol valerate-alone therapy group. The endometrial fibrosis area was $61 \%$ decreased in the combination therapy group compared with in the oestradiol valerate-alone therapy group (Fig. 1). In addition, the expression levels of the fibrosis markers (COL1A1, $\alpha$-SMA and fibronectin) in tissues were analysed with IHC staining. COL1A1, $\alpha$-SMA and fibronectin exhibited strong staining in the preoperative endometrial tissues from the two groups. These 3 proteins were mildly expressed or were undetected in the postoperative tissues. However, 3 proteins levels were expressed less in the combination therapy group compared with in the oestradiol valerate-alone therapy group (Fig. 2). The levels of 3 fibrotic markers were examined at the mRNA level by RT-qPCR and at the protein level by western blot analysis in the pre- and postoperative tissues. There was no significant difference in preoperative expression levels between the two groups. However, the expression levels of the two groups were significantly decreased following therapy compared with the preoperative levels. In samples from the combination therapy, the mRNA and relative protein expression levels of $\alpha$-SMA, COL1A1 and fibronectin were decreased compared with in the oestradiol valerate-alone therapy group (Figs. 3 and 4A-D), which were consistent with the IHC staining results. These data suggest that combination therapy with oestradiol valerate and aspirin may have a more positive effect on inhibiting endometrial fibrosis during endometrium rehabilitation in patients with IUA compared with the oestradiol valerate-alone therapy group.
Table III. Outcome measures in the study groups.

\begin{tabular}{lcc}
\hline Variable & $\begin{array}{c}\text { Group A } \\
(\mathrm{n}=26)\end{array}$ & $\begin{array}{c}\text { Group B } \\
(\mathrm{n}=28)\end{array}$ \\
\hline $\begin{array}{l}\text { Uterine length, cm } \\
\quad \text { Preoperative }\end{array}$ & $6.10 \pm 0.26$ & $6.23 \pm 0.37$ \\
$\quad$ Postoperative & $7.09 \pm 0.22^{\mathrm{b}}$ & $7.13 \pm 0.26^{\mathrm{b}}$ \\
Endometrium thickness, mm & & \\
$\quad$ Preoperative & $5.04 \pm 1.04$ & $4.96 \pm 1.04$ \\
$\quad$ Postoperative & $6.88 \pm 0.77^{\mathrm{b}}$ & $7.54 \pm 1.14^{\mathrm{a}, \mathrm{b}}$ \\
Duration of menstrual period, & & \\
days & & \\
$\quad$ Preoperative & $2.81 \pm 0.75$ & $2.71 \pm 1.08$ \\
Postoperative & $4.50 \pm 0.71^{\mathrm{b}}$ & $5.39 \pm 1.20^{\mathrm{a}, \mathrm{b}}$ \\
Menstrual volume, ml & & \\
Preoperative & $30.77 \pm 9.56$ & $31.61 \pm 9.72$ \\
Postoperative & $55.77 \pm 8.45^{\mathrm{b}}$ & $63.57 \pm 12.39^{\mathrm{a}, \mathrm{b}}$ \\
Postoperative adhesion cases, & & \\
$\mathrm{n}$ (\%) & & \\
$\quad$ No & $9(34.6)$ & $21(75.0)^{\mathrm{a}}$ \\
Mild & $7(26.9)$ & $3(10.7)$ \\
Moderate & $5(19.2)$ & $2(7.1)$ \\
Severe & $5(19.2)$ & $2(7.1)$ \\
Postoperative average AFS & $5.62 \pm 2.42$ & $3.43 \pm 1.73^{\mathrm{a}}$ \\
score & & \\
\hline
\end{tabular}

Data are presented as the mean \pm standard deviation or $\mathrm{n}(\%) .{ }^{\mathrm{a}} \mathrm{P}<0.05$ vs. group $\mathrm{A} ;{ }^{\mathrm{b}} \mathrm{P}<0.05$ vs. preoperative values within the same group. Group A, oestradiol valerate therapy; Group B, combination therapy of aspirin and oestradiol valerate therapy; AFS, American Fertility Society.

Aspirin inhibits endometrial fibrosis by suppressing the TGF- 31 -Smad2/Smad3 pathway in intrauterine adhesions. In order to understand the molecular mechanisms of aspirin inhibition of endometrial fibrosis, the present study examined changes in TGF- $\beta 1$, Smad2, p-Smad2, Smad 3 and p-Smad 3 in endometrial tissues both prior to and following treatment. The IHC and western blot analysis results revealed that TGF- $\beta 1$, p-Smad 2 and p-Smad 3 were expressed at high levels in preoperative endometrial tissues, but that this level decreased following surgery in the two groups. Combination therapy with aspirin and oestradiol valerate resulted in a significant inhibition of TGF- $\beta 1, \mathrm{p}-\mathrm{Smad} 2$ and $\mathrm{p}-\mathrm{Smad} 3$ expression levels in the uterine tissues compared with oestradiol valerate alone. However, no significant changes in total Smad2 and Smad3 levels were observed (Figs. 4A, E-G and 5). These data indicated that aspirin may inhibit postoperative endometrial fibrosis by suppressing the TGF- $\beta 1-\operatorname{Smad} 2 / \mathrm{Smad} 3$ pathway in IUAs.

\section{Discussion}

IUAs are a major health problem that lead to abnormal menstrual patterns, repeated spontaneous abortions and female infertility. 

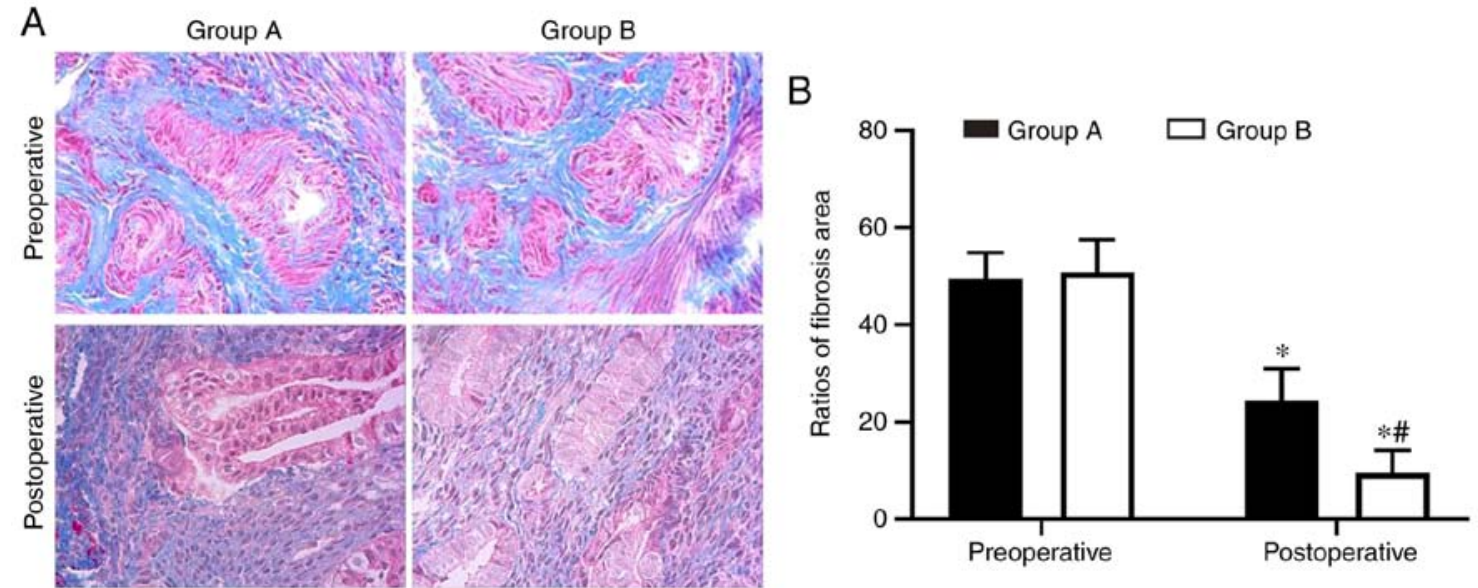

Figure 1. Aspirin inhibits endometrium fibrosis. (A) Fibrosis was detected by Masson's Trichrome staining. Magnification, x200. Fibrotic areas were stained blue. (B) Fibrosis was significantly decreased in endometrium tissues in the aspirin and oestradiol valerate therapy group (group B; $n=28$ ) compared with the oestradiol valerate therapy group (group $\mathrm{A} ; \mathrm{n}=26$ ) following treatment. ${ }^{*} \mathrm{P}<0.05$ vs. preoperative; ${ }^{"} \mathrm{P}<0.05$ vs. group $\mathrm{A}$.

A

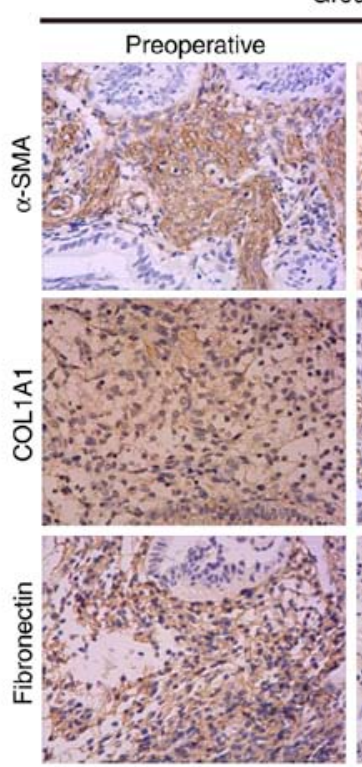

B

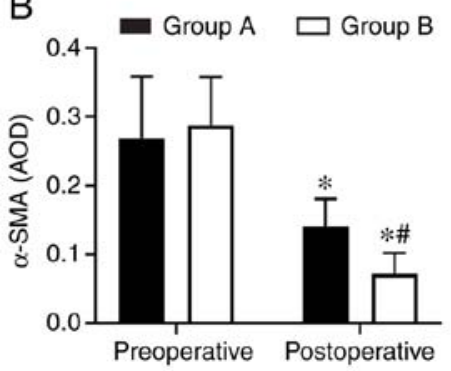

Group A
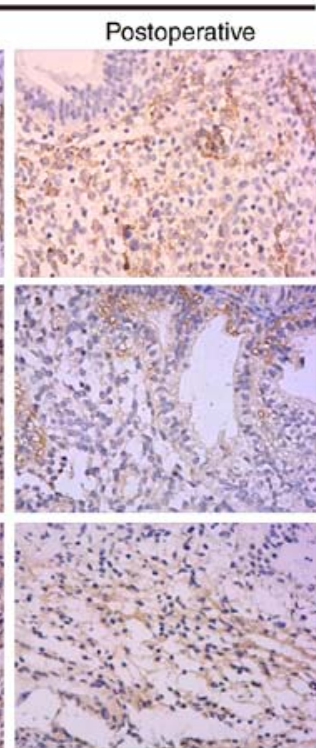

C

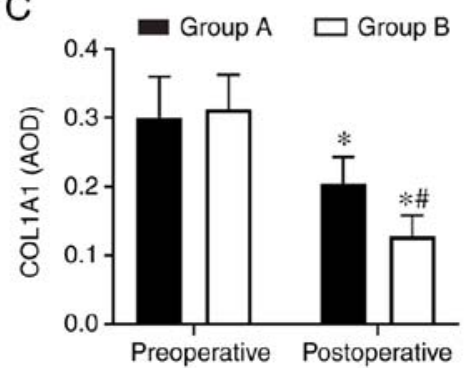

Group B
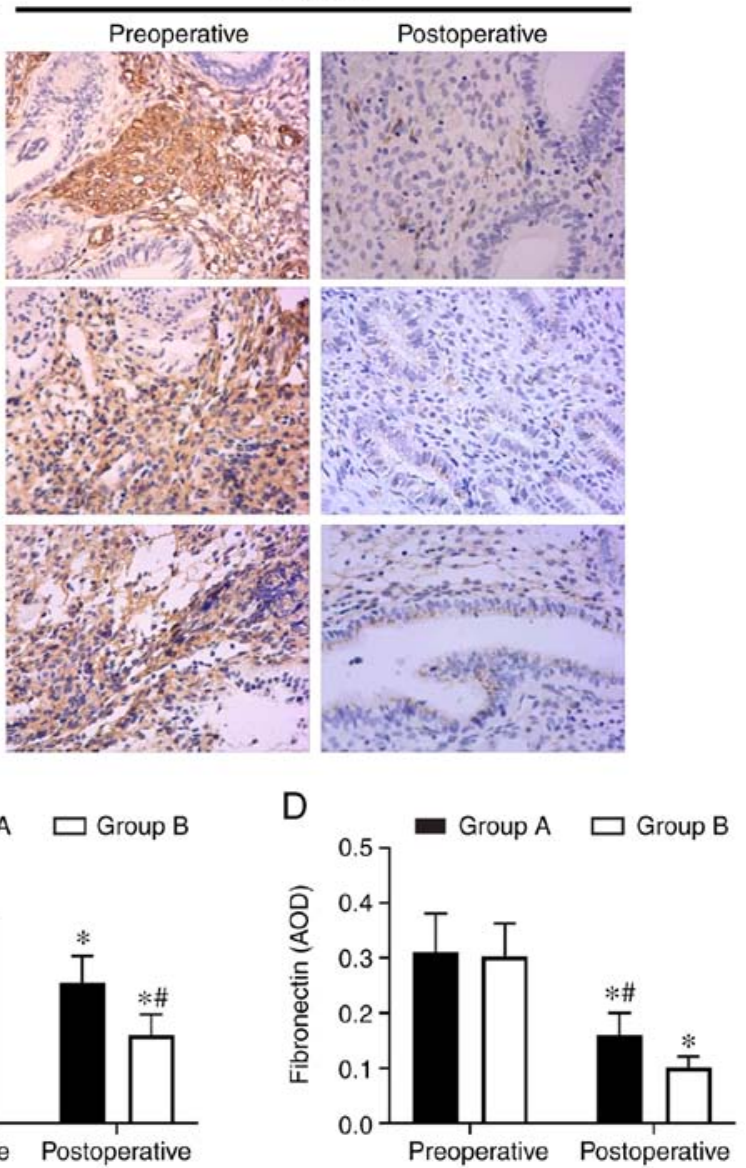

Figure 2. Aspirin inhibits the expression of fibrosis markers COL1A1, $\alpha$-SMA and fibronectin in endometrial tissues. (A) The expression levels of COL1A1, $\alpha$-SMA and fibronectin were detected by immunohistochemistry. Magnifcation, x200. (B-D) The levels of fibrosis markers (B) COL1A1, (C) $\alpha$-SMA and (D) fibronectin were significantly inhibited in endometrium tissues in the aspirin and oestradiol valerate therapy group (group B; $\mathrm{n}=28$ ) compared with the oestradiol valerate therapy group (group $\mathrm{A} ; \mathrm{n}=26$ ) following treatment. " $\mathrm{P}<0.05$ vs. preoperative. ${ }^{\text {" }} \mathrm{P}<0.05$ vs. group $\mathrm{A}$. COL1A1, collagen type I $\alpha 1$ chain; $\alpha$-SMA, $\alpha$-smooth muscle actin.

In the endometrial tissues of patients with IUAs, a large amount of fibrous tissue replaces the endometrium, destroying the basal layer of the endometrium, decreasing the blood vessels in the tissue and decreasing the perfusion of oestrogen and progesterone, further aggravating intrauterine adhesions (5). At present, the treatment goals for IUAs are primarily to restore the normal shape and volume of the uterine cavity and prevent adhesion recurrence and treatment-associated symptoms, 

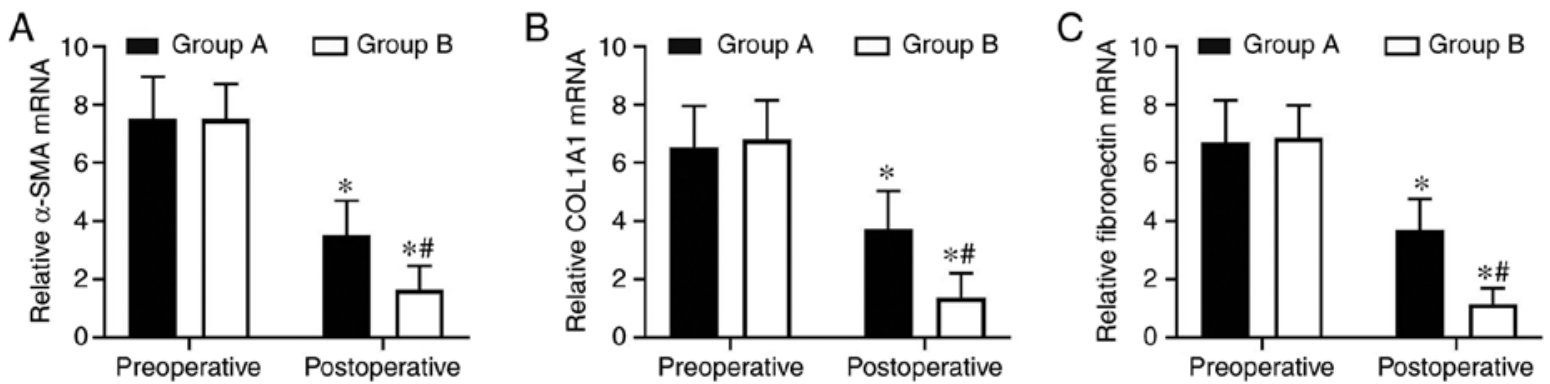

Figure 3. Aspirin inhibits the mRNA expression of COL1A1, $\alpha$-SMA and fibronectin in endometrial tissues. (A-C) The expression levels of (A) COL1A1, (B) $\alpha$-SMA and (C) fibronectin in endometrial tissues were determined at the mRNA level by reverse transcription quantitative polymerase chain reaction analysis. The relative quantity of mRNA was calculated by comparison against the internal control, GAPDH. The mRNA levels of COL1A1, $\alpha$-SMA and fibronectin were significantly inhibited in the endometrium tissues within the aspirin and oestradiol valerate therapy group (group B; $\mathrm{n}=28$ ) compared with the oestradiol valerate therapy group (group $\mathrm{A} ; \mathrm{n}=26$ ) following treatment. ${ }^{\text {}} \mathrm{P}<0.05$ vs. preoperative. ${ }^{~} \mathrm{P}<0.05$ vs. group $\mathrm{A}$. COL $1 \mathrm{~A} 1$, collagen type $\mathrm{I} \alpha 1$ chain; $\alpha$-SMA, $\alpha$-smooth muscle actin.
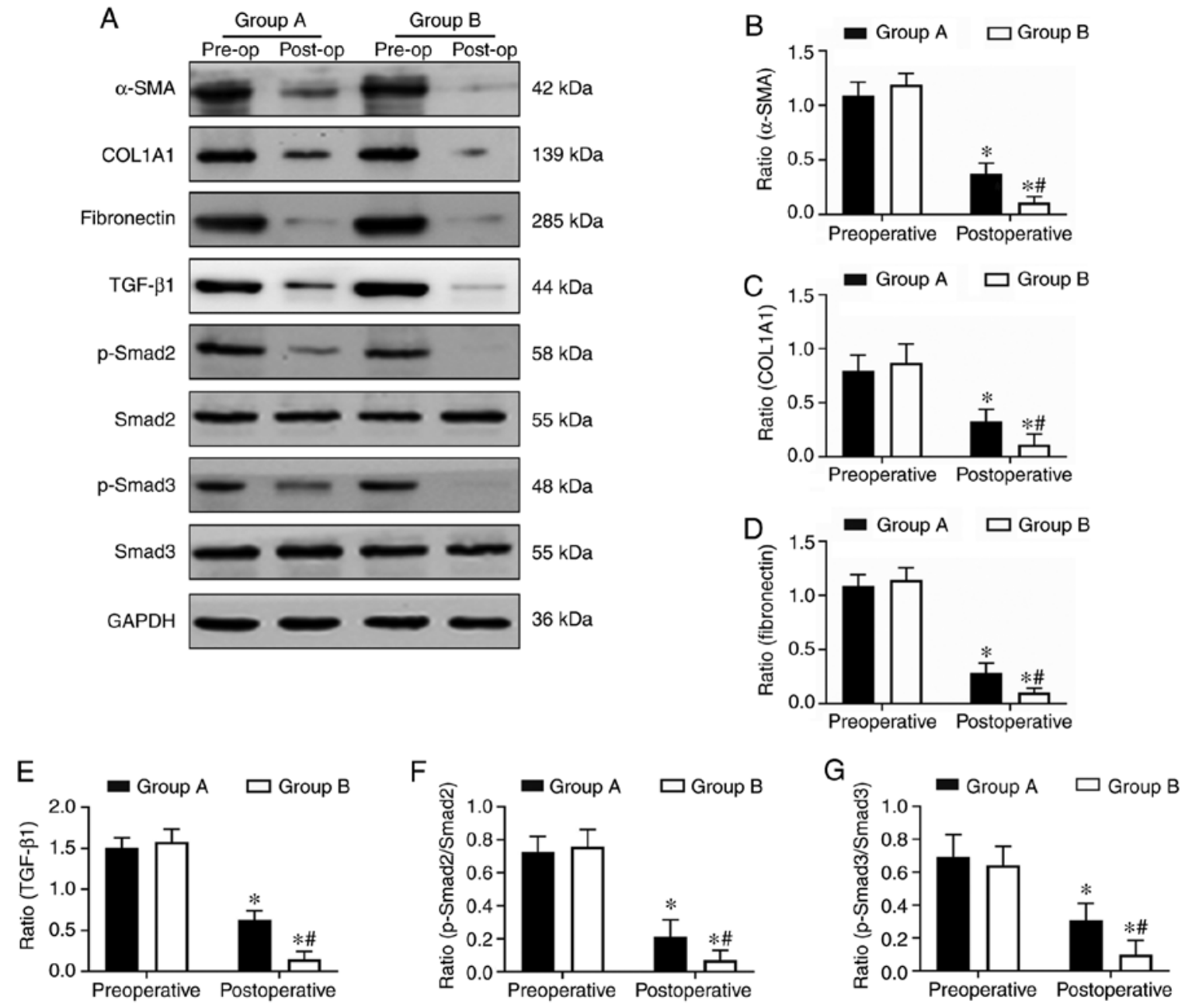

Figure 4. Aspirin inhibits the protein expression levels of COL1A1, $\alpha$-SMA, fibronectin and the TGF- $\beta 1$-Smad2/Smad3 pathway in endometrial tissues. (A) The expression levels of COL1A1, $\alpha$-SMA, fibronectin, TGF- $\beta 1$, Smad2, $\mathrm{p}$-Smad2, Smad3 and p-Smad3 in endometrial tissues were determined at the protein level by western blot analysis. (B-E) The relative protein quantities (COL1A1, $\alpha$-SMA, fibronectin, TGF- $\beta 1$ ) were calculated by comparison against the internal control, GAPDH. ( $\mathrm{F}$ and G) The relative quantity of p-Smad2 and p-Smad3 were calculated by comparison against the total Smad2 and Smad3, respectively. The protein levels of the fibrosis markers (COL1A1, $\alpha$-SMA, fibronectin), TGF- $\beta 1, \mathrm{p}$-Smad2/Smad 2 and p-Smad $3 / \mathrm{Smad} 3$, were significantly inhibited in the endometrium tissues in the aspirin and oestradiol valerate therapy group (group $B ; n=8$ ) compared with the oestradiol valerate therapy group (group $\mathrm{A} ; \mathrm{n}=8$ ) following treatment. ${ }^{*} \mathrm{P}<0.05$ vs. preoperative. ${ }^{\prime \prime} \mathrm{P}<0.05$ vs. group A. Pre-op, preoperative; post-op, postoperative; COL1A1, collagen type I $\alpha 1$ chain; $\alpha$-SMA, $\alpha$-smooth muscle actin; TGF- $\beta 1$, transforming growth factor $\beta 1$; p-, phosphorylated.

including infertility and amenorrhea (26). Although intrauterine adhesions can be improved to a certain extent through surgery and treatment, there is currently no effective strategy to prevent disease recurrence following TCRA surgery. 
A

A Group A
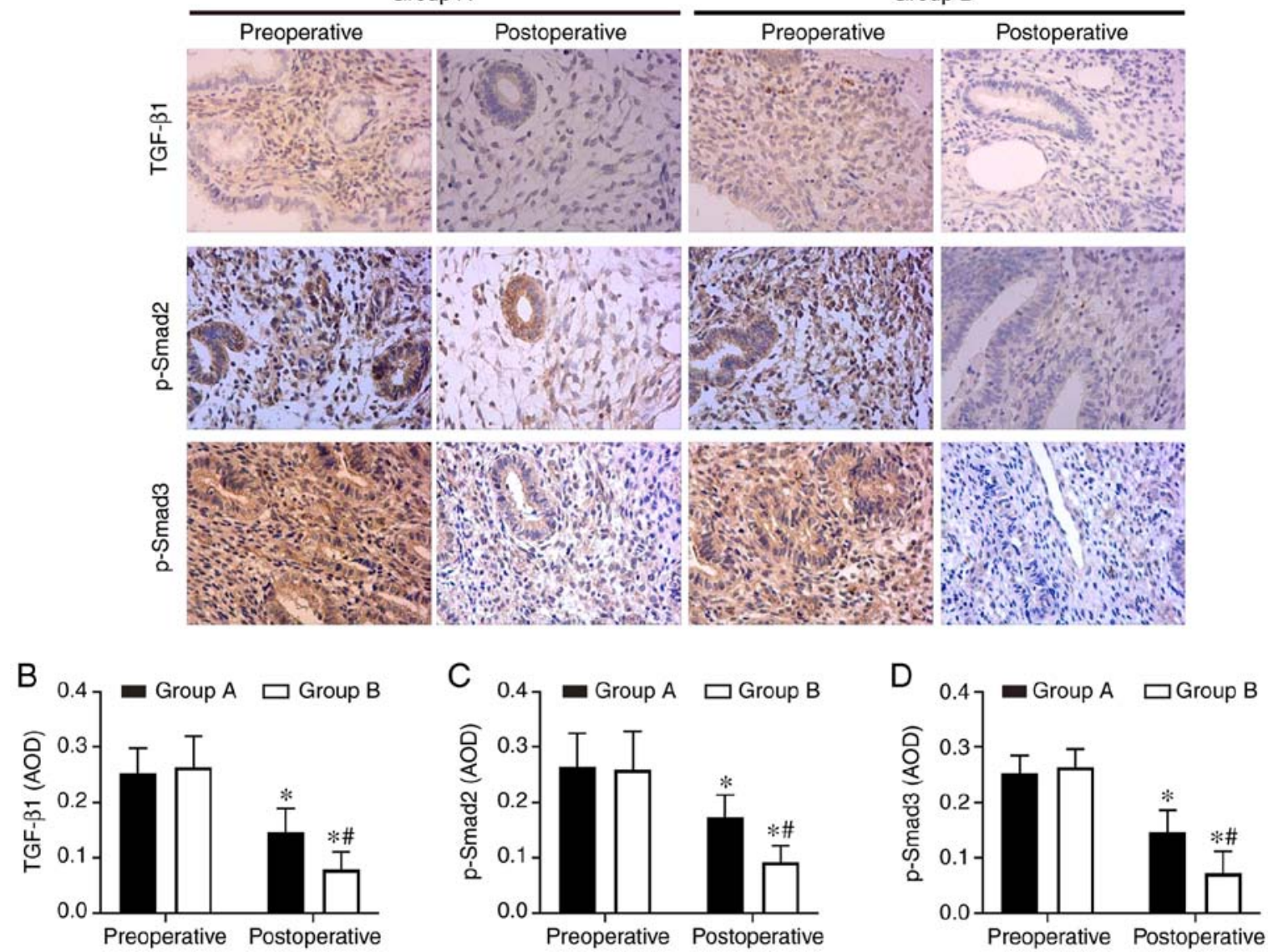

Figure 5. Aspirin inhibits the expression of the TGF- $\beta 1-\mathrm{Smad} 2 / \mathrm{Smad} 3$ pathway proteins in endometrial tissues. (A) The expression levels of TGF- $\beta 1, \mathrm{p}-\mathrm{Smad} 2$ and p-Smad3 were detected by immunohistochemistry. Magnifcation, x200. (B-D) TGF- $\beta 1$, p-Smad 2 and p-Smad3 were significantly inhibited in the endometrium tissues in the aspirin and oestradiol valerate therapy group (group $B ; n=28$ ) compared with the oestradiol valerate therapy group (group $A ; n=26$ ) following treatment. ${ }^{*} \mathrm{P}<0.05$ vs. preoperative, ${ }^{\#} \mathrm{P}<0.05$ vs. group $\mathrm{A}$. TGF- $\beta 1$, transforming growth factor $\beta 1$; $\mathrm{p}$-, phosphorylated.

Extensive studies have been performed regarding this issue, including oral oestrogen (27), transdermal oestrogen gel (28), placement of an intrauterine balloon (29) or intrauterine device (30), intrauterine injection of sodium hyaluronate (31), amniotic membrane transplantation (32) and other methods following TRCA surgery. In order to obtain improved therapeutic effects in patients with moderate and severe IAUs, it is necessary to use oestrogen combined with other adjuvant therapy following TCRA (33). Bosteels et al (34) statistically analysed the data on drug therapy to prevent adhesion recurrence and treatment of infertility following TCRA surgery prior to June 2017, and suggested that it remains uncertain whether anti-adhesion therapy improves the critical reproductive outcome or decreases the severity of IUAs. Of course, due to the blind manner in which the participants and personnel were involved, as well as the risk of serious bias associated with indirectness and inaccuracy, the overall quality of the evidence is low. Conversely, although these treatments may improve symptoms to a certain extent, the overall treatment effect remains unsatisfactory.

Aspirin is one of the most widely used drugs in the world. Aspirin was originally used primarily in patients with antipyretic and analgesic diseases. At present, there is increasing evidence that aspirin has a preventative effect in colorectal cancer and other types of cancer (35). Recent studies have suggested that aspirin is more effective in preventing adhesion recurrence following TCRA surgery, as it can increase endometrial angiogenesis (28). A prospective study revealed that aspirin combined with an intrauterine balloon and intrauterine device significantly increased endometrial thickness following TCRA surgery, and AFS scores and menstrual scores also improved significantly (23). Chi et al (28) demonstrated that compared with transdermal oestrogen alone therapy, transdermal oestrogen combined with aspirin following TCRA surgery resulted in an improved effect on increasing the endometrial thickness, increasing menstrual flow, preventing adhesion recurrence and improving endometrial receptivity, which may be associated with increased uterus intimal angiogenesis and decreased resistant index and pulsatility index in uterine arteries. The endometrial repair time following TCRA surgery is usually $\sim 2$ months (36). Therefore, all patients in the present study were reviewed 2 months after TCRA to assess for endometrial recovery. The results of the present study revealed that patients receiving aspirin combined with oestrogen and progesterone following TCRA exhibited positive effects regarding improved menstruation, increased endometrial thickness and improved AFS scores compared with patients receiving single oestrogen and progesterone.

Although there are some studies on the application of aspirin as an anti-fibrosis treatment, the specific anti-fibrosis 
mechanism of aspirin remains unclear. Aspirin decreases cardiac interstitial fibrosis caused by pressure overload in transverse aortic constriction (TAC) mice, which may be associated with the inhibition of the $\mathrm{p}$-Erk $1 / 2$ and $\mathrm{p}-\mathrm{Akt} / \beta$-catenin signalling pathways by aspirin, thereby decreasing the expression levels of $\alpha$-SMA and collagen I (37). A previous study revealed that aspirin alleviated cardiac fibrosis in mice by inhibiting autophagy (22). In a rat liver fibrosis model, aspirin improved the degree of liver fibrosis in a dose-dependent manner (21), but the underlying molecular mechanism remains unclear. Endometrial fibrosis is considered to be a key pathological event in the development of IUAs (38). Previous studies have demonstrated that aspirin may decrease fibrotic markers, including TGF- $\beta$, connective tissue growth factor (CTGF), collagen I and collagen III, in endometrial tissue (28), but the specific mechanism of action remains unclear. The results of the present study revealed that the expression of COL1A1, $\alpha$-SMA and FN in the endometrium of the aspirin group was decreased, thereby indicating an active role in the anti-fibrosis process.

TGF- $\beta 1$ is considered to be a key mediator of fibrosis. It serves a vital role in regulating wound healing and tissue repair. Activation and overexpression of TGF- $\beta 1$ are associated with tissue scarring and fibrosis (39). TGF- $\beta 1$ activates both classical (Smad-based) signaling pathways and non-classical (non-Smad-based) signaling pathways, leading to the activation of myofibroblasts, overproduction of extracellular matrix (ECM) and inhibition ECM degradation (40). In Smad-based signaling pathways, TGF- $\beta 1$ binds to the TGF- $\beta 1$ receptor on the cell membrane to form a ligand-receptor complex, which in turn binds, phosphorylates and activates Smad2 and Smad3. Smad4 then binds activated Smad2/3, and the complex enters the nucleus and interacts with transcription factors to induce transcription of profibrotic molecules, including $\alpha$-SMA, collagen I, fibronectin and tissue inhibitor of matrix metalloproteinases (TIMP) (40). Conversely, Smad3 may induce transcription of fibrotic fs (miRNAs) and long non-coding RNAs (lncRNAs), while indirectly inhibiting transcription of anti-fibrotic miRNAs. In addition, Smad3 can increase the transcription of fibrotic molecules by affecting epigenetic modifications of DNA and histones (40). There have been advances in the progress of various fibrotic diseases treatment options that target the TGF- $\beta 1 /$ Smad signaling pathway, including idiopathic pulmonary fibrosis and renal fibrosis $(41,42)$. The TGF- $\beta$ type I receptor kinase small molecule inhibitor EW-7197 inhibits the TGF- $\beta 1 / \mathrm{Smad} 2 / 3$ signalling pathway and has potential for anti-fibrotic therapy (43). Suberoylanilide hydroxamic acid, an inhibitor of histone deacetylases that may decrease the levels of TGF- $\beta 1$, CTGF, $\alpha$-SMA, $p$-Smad $2 / 3$, can alleviate rat liver fibrosis by suppressing TGF- $\beta 1$ signaling (44). These data indicate that targeting the TGF- $\beta 1$-Smad signaling pathway is a potential avenue of anti-fibrotic therapy.

TGF- $\beta 1$ has a specific role in the occurrence and progression of IUAs, and the expression levels of TGF- $\beta 1$ and Smad3 are positively correlated with the severity of IUAs $(15,45)$. Studies have revealed that miRNA-29b and miRNA-326 may improve endometrial fibrosis in primary human endometrial stromal cells, and can significantly decrease COL1A1, $\alpha$-SMA and FN expression by inhibiting the TGF- $\beta 1 /$ Smad signalling pathway $(14,46)$. In order to investigate the potential mechanism underlying the anti-fibrosis effect of aspirin, the present study examined the expression levels of TGF- $\beta 1$, Smad2 and Smad3 in the endometrial tissues of two groups. The results of the present study demonstrated that the expression levels of COL1A1, $\alpha$-SMA and FN in the endometrial tissues of the combination therapy group were decreased compared with those in the oestradiol valerate group. Aspirin may inhibit the expression of TGF- $\beta 1, \mathrm{p}-\mathrm{Smad} 2$ and $\mathrm{p}-\mathrm{Smad} 3$ by TGF- $\beta 1$-Smad-based signaling pathways in endometrial tissues, which may be one of the mechanisms by which aspirin improves endometrial fibrosis.

It is worth noting that combination therapy with oestrogen and aspirin may improve the rate of pregnancy in patients with IUA (28). However, an additional study indicated that there was no significant improvement in the pregnancy rate and live birth rate following additional low dose aspirin (23). Different results may be associated with the different doses of aspirin, which suggests that aspirin serves a positive role in improving the pregnancy outcome of patients with IUAs. The appropriate dose of aspirin required for endometrial repair following TCRA recovery requires a continuous follow-up study. In addition, obstetric complications should be observed, including placentation issues, prematurity and postpartum hysterectomy following treatment for IUAs (47). For example, intrauterine adhesions may lead to placenta accreta and the risk of severe postpartum haemorrhage (48). However, to the best of our knowledge, whether aspirin may decrease the obstetric complications with IUAs following surgery has not yet been described in the literature and requires long-term follow-up observations.

One potential limitation of the present study is that there was a relatively small number of cases and a short follow-up time period. A larger sample size is required in order to assess the appropriate dose and the duration of aspirin treatment, and the effect on pregnancy outcomes and obstetric complications, which will form the basis for future studies. In addition, endometrial tissue samples from patients with IUAs are rare and difficult to obtain: A small amount of endometrial tissue can be valuable for endometrial repair in IUAs, and certain patients with intrauterine adhesions are reluctant to participate in research for this reason. Animal models of intrauterine adhesion will be used in future studies to further investigate the mechanism of anti-endometrial fibrosis in aspirin.

In conclusion, aspirin combined with oestradiol valerate exhibited an improved effect on increasing endometrial thickness and improving menstrual and AFS scores following TCRA compared with oestradiol valerate alone. This result reveals that aspirin inhibits endometrial fibrosis by suppressing the TGF- $\beta 1$-Smad2/Smad3 pathway in IUAs.

\section{Acknowledgements}

Not applicable.

\section{Funding}

The present study was supported by the Faculty Development Grants From Xiangyang No. 1 People's Hospital (grant no. 28) and the Natural Science Foundation of Hubei Province of China (grant nos. 2019AHB068 and 2018CFB701). 


\section{Availability of data and materials}

The datasets used and/or analysed during the present study are available from the corresponding author on reasonable request.

\section{Authors' contributions}

$\mathrm{ZZ}$ and SL were responsible for protocol/project development and manuscript writing. JD, SY, ZX, HG and HX performed data management and analysis. WZ was involved in protocol/project development and supervision. MS reviewed the final manuscript and data analysis. All authors read and approved the final manuscript.

\section{Ethics approval and consent to participate}

The present study was reviewed and approved by the Ethics Committee of Xiangyang No. 1 People's Hospital, Hubei University of Medicine (approval no. 2018KYLL), and written informed consent was obtained from all patients prior to their inclusion in the study.

\section{Patient consent for publication}

The patients or their guardians have provided written informed consent for the publication of any associated data and accompanying images.

\section{Competing interests}

The authors declare that they have no competing interests.

\section{References}

1. Asherman JG: Amenorrhoea traumatica (atretica). J Obstet Gynaecol Br Emp 55: 23-30, 1948.

2. Healy MW, Schexnayder B, Connell MT, Terry N, DeCherney AH, Csokmay JM, Yauger BJ and Hill MJ: Intrauterine adhesion prevention after hysteroscopy: A systematic review and meta-analysis. Am J Obstet Gynecol 215: 267-275.e7, 2016.

3. Deans R and Abbott J: Review of intrauterine adhesions. J Minim Invasive Gynecol 17: 555-569, 2010.

4. Bhandari S, Bhave P, Ganguly I, Baxi A and Agarwal P: Reproductive outcome of patients with Asherman's syndrome: A SAIMS experience. J Reprod Infertil 16: 229-235, 2015.

5. Yu D, Wong YM, Cheong Y, Xia E and Li TC: Asherman syndrome-one century later. Fertil Steril 89: 759-779, 2008.

6. Bottinger EP: TGF-beta in renal injury and disease. Semin Nephrol 27: 309-320, 2007.

7. Derynck $R$ and Zhang YE: Smad-dependent and Smad-independent pathways in TGF-beta family signalling. Nature 425: 577-584, 2003.

8. Liu G, Ma C, Yang H and Zhang PY: Transforming growth factor beta and its role in heart disease. Exp Ther Med 13: 2123-2128, 2017.

9. Shevchenko OP, Kurabekova RM and Tsirulnikova OM: The role of transforming growth factor beta1 under diseases of liver. Klin Lab Diagn 62: 161-164, 2017 (In Russian).

10. Zhou LL, Wang M, Liu F, Lu YZ, Song LJ, Xiong L, Xiang F, He XL, Shuai SY, Xin JB, et al: Cigarette smoking aggravates bleomycin-induced experimental pulmonary fibrosis. Toxicol Lett 303: 1-8, 2019.

11. Liu P, Zhu L, Zou G and Ke H: Matrine suppresses pancreatic fibrosis by regulating TGF- $\beta /$ Smad signaling in rats. Yonsei Med J 60: 79-87, 2019.

12. Meng XM, Tang PM, Li J and Lan HY: TGF- $\beta /$ Smad signaling in renal fibrosis. Front Physiol 6: 82, 2015.
13. Sisto M, Lorusso L, Ingravallo G, Tamma R, Ribatti D and Lisi S: The TGF- $\beta 1$ signaling pathway as an attractive target in the fibrosis pathogenesis of sjogren's syndrome. Mediators Inflamm 2018: 1965935, 2018.

14. Ning J, Zhang H and Yang H: MicroRNA 326 inhibits endometrial fibrosis by regulating TGF $\beta 1 / \mathrm{Smad} 3$ pathway in intrauterine adhesions. Mol Med Rep 18: 2286-2292, 2018.

15. Salma U, Xue M, Ali Sheikh MS, Guan X, Xu B, Zhang A Huang $L$ and $X u$ D: Role of transforming growth factor- $\beta 1$ and smads signaling pathway in intrauterine adhesion. Mediators Inflamm 2016: 4158287, 2016.

16. Patrono C: Aspirin as an antiplatelet drug. N Engl J Med 330: 1287-1294, 1994

17. Vonkeman HE and van de Laar MA: Nonsteroidal anti-inflammatory drugs: Adverse effects and their prevention. Semin Arthritis Rheum 39: 294-312, 2010.

18. Albandar HJ, Markert R and Agrawal S: The relationship between aspirin use and mortality in colorectal cancer. J Gastrointest Oncol 9: 1133-1137, 2018

19. Rosenberg K and Mechcatie E: Low-dose aspirin use associated with lower ovarian cancer risk. Am J Nurs 119: 50, 2019.

20. Jiang ZG, Feldbrügge L, Tapper EB, Popov Y, Ghaziani T, Afdhal N, Robson SC and Mukamal KJ: Aspirin use is associated with lower indices of liver fibrosis among adults in the United States. Aliment Pharmacol Ther 43: 734-743, 2016.

21. Li CJ, Yang ZH, Shi XL and Liu DL: Effects of aspirin and enoxaparin in a rat model of liver fibrosis. World J Gastroenterol 23: 6412-6419, 2017.

22. Liu PP, Liu HH, Sun SH, Shi XX, Yang WC, Su GH and Zhao J: Aspirin alleviates cardiac fibrosis in mice by inhibiting autophagy. Acta Pharmacol Sin 38: 488-497, 2017.

23. Chen Y, Liu L, Luo Y, Chen M, Huan Y and Fang R: Effects of aspirin and intrauterine balloon on endometrial repair and reproductive prognosis in patients with severe intrauterine adhesion: A prospective cohort study. Biomed Res Int 2017: 8526104, 2017.

24. The American Fertility Society classifications of adnexal adhesions, distal tubal occlusion, tubal occlusion secondary to tubal ligation, tubal pregnancies, mullerian anomalies and intrauterine adhesions. Fertil Steril 49: 944-955, 1988.

25. Livak KJ and Schmittgen TD: Analysis of relative gene expression data using realtime quantitative PCR and the 2(-Delta DeltaC(T)) method. Methods 25: 402-408, 2001.

26. Zupi E, Centini G and Lazzeri L: Asherman syndrome: An unsolved clinical definition and management. Fertil Steril 104: 1380-1381, 2015

27. Liu L, Huang X, Xia E, Zhang X, Li TC and Liu Y: A cohort study comparing $4 \mathrm{mg}$ and $10 \mathrm{mg}$ daily doses of postoperative oestradiol therapy to prevent adhesion reformation after hysteroscopic adhesiolysis. Hum Fertil (Camb) 22: 191-197, 2018.

28. Chi Y, He P, Lei L, Lan Y, Hu J, Meng Y and Hu L: Transdermal estrogen gel and oral aspirin combination therapy improves fertility prognosis via the promotion of endometrial receptivity in moderate to severe intrauterine adhesion. Mol Med Rep 17: 6337-6344, 2018.

29. Zhu R, Duan H, Gan L and Wang S: Comparison of intrauterine suitable balloon and foley balloon in the prevention of adhesion after hysteroscopic adhesiolysis. Biomed Res Int 2018: 9494101, 2018.

30. Lin XN, Zhou F, Wei ML, Yang Y, Li Y, Li TC and Zhang SY: Randomized, controlled trial comparing the efficacy of intrauterine balloon and intrauterine contraceptive device in the prevention of adhesion reformation after hysteroscopic adhesiolysis. Fertil Steril 104: 235-240, 2015.

31. Bosteels J, Weyers S, Mol BW and D'Hooghe T: Anti-adhesion barrier gels following operative hysteroscopy for treating female infertility: A systematic review and meta-analysis. Gynecol Surg 11: 113-127, 2014.

32. Amer MI, Abd-El-Maeboud KH, Abdelfatah I, Salama FA and Abdallah AS: Human amnion as a temporary biologic barrier after hysteroscopic lysis of severe intrauterine adhesions: Pilot study. J Minim Invasive Gynecol 17: 605-611, 2010.

33. Johary J, Xue M, Zhu X, Xu D and Velu PP: Efficacy of estrogen therapy in patients with intrauterine adhesions: Systematic review. J Minim Invasive Gynecol 21: 44-54, 2014.

34. Bosteels J, Weyers S, D'Hooghe TM, Torrance H, Broekmans FJ, Chua SJ and Mol BWJ: Anti-adhesion therapy following operative hysteroscopy for treatment of female subfertility. Cochrane Database Syst Rev 11: CD011110, 2017.

35. Montinari MR, Minelli S and De Caterina R: The first 3500 years of aspirin history from its roots-A concise summary. Vascul Pharmacol 113: 1-8, 2019. 
36. Yang JH, Chen MJ, Chen CD, Chen SU, Ho HN and Yang YS: Optimal waiting period for subsequent fertility treatment after various hysteroscopic surgeries. Fertil Steril 99: 2092-2096.e3, 2013.

37. Li X, Wang G, QiLi M, Liang H, Li T, E X, Feng Y, Zhang Y, Liu X, Qian M, et al: Aspirin reduces cardiac interstitial fibrosis by inhibiting Erk1/2-Serpine2 and P-Akt signalling pathways. Cell Physiol Biochem 45: 1955-1965, 2018.

38. Zhu HY, Ge TX, Pan YB and Zhang SY: Advanced Role of Hippo Signaling in Endometrial Fibrosis: Implications for intrauterine adhesion. Chin Med J (Engl) 130: 2732-2737, 2017.

39. Branton MH and Kopp JB: TGF-beta and fibrosis. Microbes Infect 1: 1349-1365, 1999.

40. Meng XM, Nikolic-Paterson DJ and Lan HY: TGF- $\beta$ : The master regulator of fibrosis. Nature reviews. Nephrology 12: 325-338, 2016.

41. Ji Y, Dou YN, Zhao QW, Zhang JZ, Yang Y, Wang T, Xia YF Dai Y and Wei ZF: Paeoniflorin suppresses TGF- $\beta$ mediated epithelial-mesenchymal transition in pulmonary fibrosis through a Smad-dependent pathway. Acta Pharmacol Sin 37: 794-804, 2016.

42. Xu J, Yu TT, Zhang K, Li M, Shi HJ, Meng XJ, Zhu LS and Zhu LK: HGF alleviates renal interstitial fibrosis via inhibiting the TGF- $31 /$ SMAD pathway. Eur Rev Med Pharmacol Sci 22: 7621-7627, 2018

43. Park SA, Kim MJ, Park SY, Kim JS, Lee SJ, Woo HA, Kim DK, Nam JS and Sheen YY: EW-7197 inhibits hepatic, renal, and pulmonary fibrosis by blocking TGF- $\beta /$ Smad and ROS signaling. Cell Mol Life Sci 72: 2023-2039, 2015.
44. Wang Y, Zhao L, Jiao FZ, Zhang WB, Chen Q and Gong ZJ: Histone deacetylase inhibitor suberoylanilide hydroxamic acid alleviates liver fibrosis by suppressing the transforming growth factor- $\beta 1$ signal pathway. Hepatobiliary Pancreat Dis Int 17: 423-429, 2018.

45. Li J, Du S, Sheng X, Liu J, Cen B, Huang F and He Y: MicroRNA-29b inhibits endometrial fibrosis by regulating the Sp1-TGF- $\beta 1 /$ Smad-CTGF axis in a rat model. Reprod Sci 23: 386-394, 2016.

46. Li J, Cen B, Chen S and He Y: MicroRNA-29b inhibits TGF- $\beta 1$-induced fibrosis via regulation of the TGF- $\beta 1 /$ Smad pathway in primary human endometrial stromal cells. Mol Med Rep 13: 4229-4237, 2016.

47. Deans R, Vancaillie T, Ledger W, Liu J and Abbott JA: Live birth rate and obstetric complications following the hysteroscopic management of intrauterine adhesions including Asherman syndrome. Hum Reprod 33: 1847-1853, 2018.

48. Engelbrechtsen L, Langhoff-Roos J, Kjer JJ and Istre O: Placenta accreta: Adherent placenta due to Asherman syndrome. Clin Case Rep 3: 175-178, 2015.

(i) (9) This work is licensed under a Creative Common Attribution-NonCommercial-NoDerivatives 4.0 International (CC BY-NC-ND 4.0) License. 